\title{
Letter: GATA binding protein 3(+) group 2 innate lymphoid cells are present in cord blood and in higher proportions in male than in female neonates
}

Anna Forsberg, Mathias Bengtsson, Anna Eringfält, Jan Ernerudh, Jenny Mjösberg and Maria

C Jenmalm

\section{Linköping University Post Print}

\section{Tweet}

N.B.: When citing this work, cite the original article.

Original Publication:

Anna Forsberg, Mathias Bengtsson, Anna Eringfält, Jan Ernerudh, Jenny Mjösberg and Maria C Jenmalm, Letter: GATA binding protein 3(+) group 2 innate lymphoid cells are present in cord blood and in higher proportions in male than in female neonates, 2014, Journal of Allergy and Clinical Immunology, (134), 1, 228-230.

http://dx.doi.org/10.1016/j.jaci.2014.01.027

Copyright: Elsevier

http://www.elsevier.com/

Postprint available at: Linköping University Electronic Press

http://urn.kb.se/resolve?urn=urn:nbn:se:liu:diva-108375 


\section{Letter to the Editor}

\section{GATA binding protein $3^{+}$group 2 innate lymphoid cells are present in cord blood and in higher proportions in male than in female neonates}

\section{To the Editor:}

Innate lymphoid cells (ILCs) have recently gained much attention as important mediators of tissue homeostasis and inflammation. ${ }^{1}$ In contrast to other members of the ILC family, including ILC1 and ILC $3,{ }^{1}$ group 2 ILCs (ILC2) produce IL-5 and IL-13 in response to IL-25, IL-33, and thymic stromal lymphopoietin, cytokines that may be released after epithelial damage., ${ }^{2,3}$ In spite of their association with type 2 mediated inflammation in both humans and mice, ${ }^{1}$ it is not known whether ILC2 are present in cord blood or whether they are involved in subsequent allergy development. Early life events occurring during critical windows of immune development can have a longterm impact on immune-mediated diseases, and immune status at birth, in part influenced by maternal immunity, may be an intrinsic factor predisposing to allergy development. ${ }^{4}$ The aim of this study was to assess whether ILC2 are present in cord blood and whether their proportions are associated with allergy development and sex.

We report here that ILC2 are present in human cord blood (for gating strategies, see this article's Methods section and Fig E1 in the Online Repository at www.jacionline.org). Thus, we identified a population of lineage negative (Lin-) cells lacking the expression of cell surface markers associated with $\mathrm{T}$ cells (CD3, CD4, T-cell receptor (TCR) $\alpha \beta$, and TCR $\gamma \delta)$, B cells (CD19), dendritic cells (CD11c, CD123, CD303, CD1a), macrophages/monocytes (CD14), mast cells and basophils (FceR1 $\alpha$ ), and hematopoietic progenitor cells (CD34). The cells expressed CD161, CD127, and CRTH2 and lacked expression of CD56 (Fig 1, A), while CD117 was heterogeneously expressed (data not shown), as previously described in adult blood ILC2. ${ }^{2}$ It was recently discovered that human ILC2 are dependent on the expression of transcription factor GATA-3, which is important for IL-5 and IL-13 cytokine production from these cells. ${ }^{3}$ Accordingly, we found that ILC2 in peripheral blood of adults $(\mathrm{n}=7)$ and neonates $(\mathrm{n}=8)$ expressed GATA-3 in a similar way as $\mathrm{T}_{\mathrm{H}} 2$ cells, while natural killer cells (CD56dim) had low GATA-3 expression (Fig 1, $B$ and $C$ ). The GATA-3 expression was higher in neonate than in adult ILC2 $(P=.009)$, expressed as a ratio between ILC2 and natural killer cells (Fig 1,D). Speculatively, the higher GATA-3 expression could be related to the function and cytokine-producing capacity of ILC2 in cord blood. Unfortunately, no functional assays could be performed because of insufficient amounts of blood for cell isolation and culturing. However, previous studies have demonstrated the crucial function of GATA-3 in ILC2 since ectopic expression of GATA-3 in human $\operatorname{Lin}(-) \mathrm{CD} 127(+) \mathrm{CRTH} 2(-)$ cells resulted in induction of CRTH2 and the capacity to produce high amounts of type 2 cytokines in response to thymic stromal lymphopoietin plus IL$33 .^{3}$
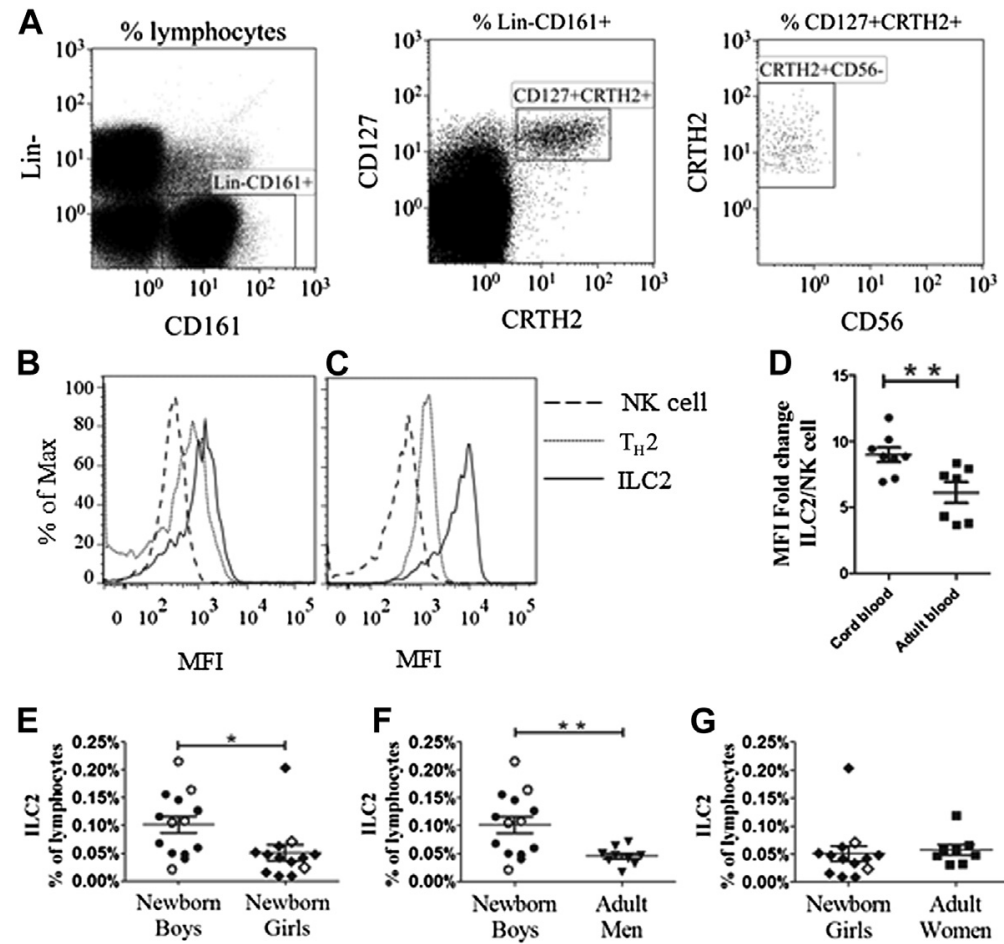

FIG 1. ILC2 are present in cord blood. A, Representative flow cytometry plots. Representative pictures of GATA-3 in ILC2, $T_{H} 2$, and NK cells (CD56dim) in adult peripheral blood (B) and cord blood (C). D, GATA-3 expression as fold change between ILC2 and NK cells (CD56dim). ILC2 proportions in boys and girls (Fig 1, D), boys and men (E), and girls and women (F). Allergic neonates are marked with unfilled circles. $\mathrm{MFI}$, Mean fluorescence intensity; NK, natural killer. 
TABLE I. Descriptive data of children included in the study

\begin{tabular}{|c|c|c|c|c|}
\hline Allergic children & Symptoms/sensitization $0-2$ y & Symptoms/sensitization 2-6 y & Sex & $\begin{array}{c}\text { Maternal atopy } \\
\text { (symptoms/sensitization) }\end{array}$ \\
\hline 1 & & ARC, SPT + birch, timothy, Phinf,+ Phad + & Boy & ARC/positive \\
\hline 2 & AD, SPT + egg, milk, Phinf + & $\mathrm{AB}, \mathrm{U}$ & Boy & No/negative \\
\hline 3 & AD, AB, U, SPT + egg, Phinf + & $\mathrm{AD}, \mathrm{AB}, \mathrm{SPT}+$, timothy, Phinf + , Phad + & Girl & ARC/positive \\
\hline 4 & AD, SPT + egg, milk, Phinf + & ARC, SPT + , birch, timothy, cat, Phinf,+ Phad + & Boy & No/negative \\
\hline 5 & $\mathrm{AD}, \mathrm{AB}, \mathrm{SPT}+$ egg, Phinf + & $\mathrm{AD}, \mathrm{AB}, \mathrm{SPT}+$ egg, Phinf,+ Phad + & Girl & No/positive \\
\hline 6 & Phinf + & $\mathrm{ARC}, \mathrm{SPT}+$ & Boy & AB/negative \\
\hline 7 & AD, Phinf + & - & Boy & ARC/negative \\
\hline 8 & - & - & Boy & ARC, U/positive \\
\hline 9 & - & - & Girl & No/negative \\
\hline 10 & - & - & Girl & No/negative \\
\hline 11 & - & - & Girl & No/negative \\
\hline 12 & - & - & Boy & No/positive \\
\hline 13 & - & - & Boy & $\mathrm{AB} /$ negative \\
\hline 14 & - & - & Boy & No/negative \\
\hline
\end{tabular}

$A B$, Asthma bronchiale; $A D$, atopic dermatitis; $A R C$, allergic rhinoconjunctivitis; Phad, Phadiatop test; Phinf, Phadiatop Infant test; $S P T$, skin prick test; $U$, urticaria.

Because a more pronounced $\mathrm{T}_{\mathrm{H}} 2$ deviation is suggested to precede the development of allergic disease ${ }^{4}$ and ILC2 have been implicated to be involved in allergic responses, ${ }^{1,2}$ we investigated whether high ILC2 proportions in cord blood could predict the development of allergic disease. However, no differences were detected in cord blood between children who later developed allergic diseases and those who remained nonallergic up to the age of 6 years (Table I) (percentages of ILC2 among lymphocytes: mean, $0.10 \pm 0.03, \mathrm{n}=7$, and mean $0.09 \pm 0.02, \mathrm{n}=7$, respectively). Neither did maternal atopy affect the ILC2 proportions (percentages of ILC2 among lymphocytes: mean, $0.09 \pm$ $0.02, \mathrm{n}=12$, and mean $0.07 \pm 0.01, \mathrm{n}=15$, in children of atopic and nonatopic mothers, respectively). Our observations suggest that cord blood ILC2 proportions are not related to allergy development, although this should be confirmed in a larger study. The mean ILC2 proportions were very similar in children developing allergy or staying healthy according to our strict criteria, however. Previously, it has been shown that children who later develop allergic disease have a more pronounced $\mathrm{T}_{\mathrm{H}} 2$ deviation already at birth, ${ }^{4}$ with enhanced circulating $\mathrm{T}_{\mathrm{H}} 2$-associated chemokine levels, ${ }^{5}$ which would suggest a role for increased ILC2 proportions in neonates later developing allergies. However, the involvement of ILC2 in allergic disease has primarily been observed at effector sites, that is, at mucosal surfaces. ${ }^{1}$ Because no increased proportions of ILC2 could be detected in the cord blood of children later developing allergy, these cells may be recruited to and involved in the response at the effector sites rather than systemically.

Because boys are known to have a more $\mathrm{T}_{\mathrm{H}}$ 2-deviated immunity ${ }^{6,7}$ and an increased susceptibility to $\mathrm{T}_{\mathrm{H}} 1$-dependent infections early in life compared with girls, ${ }^{6}$ we were interested to see whether sex was associated with the ILC2 proportions at birth. Notably, newborn boys $(\mathrm{n}=14)$ had significantly higher proportion of ILC2 than did newborn girls (n $=13, P=.02$; Fig $1, E)$. There were no differences between adult men $(\mathrm{n}=9)$ and women $(\mathrm{n}=8)$, but boys had significantly higher proportions of ILC2 than did men $(P=.009$; Fig $1, F)$ while girls and women had similar ILC2 proportions (Fig 1, G). In line with these observations, sex-related differences in immune responses in children have been reported. A number of clinical studies have observed an increased prevalence of atopic diseases in boys than in girls. ${ }^{6,8}$ Likewise, females tend to have stronger $\mathrm{T}_{\mathrm{H}} 1$ responses than do males, as evident by higher levels of inflammatory markers and infection clearance. ${ }^{6,7}$ This results in not only better protection against infection but also increased susceptibility to autoimmunity. ${ }^{8}$ Allergy-related sex differences diminish at puberty, and at adult age no clear sex differences concerning allergy can be found.

This sex-based ILC2 difference was not evident in our adult population, in line with the diminished sex difference in allergic responses in adults. What remains to be elucidated in larger studies is how this sex difference relates to ILC2 frequency, function, and future allergy development. One or several shared underlying mechanisms involving both allergy and ILC2 development and function may exist but are currently unknown.

In conclusion, we demonstrated that ILC2 are present in cord blood and display a higher GATA-3 expression than in adult ILC2. The increased ILC2 proportions in male neonates could be associated with the heightened $\mathrm{T}_{\mathrm{H}} 2$ responses and susceptibility to $\mathrm{T}_{\mathrm{H}} 1$-dependent infections in boys than in girls during childhood.

Anna Forsberg, $\mathrm{MSc}^{a}$ Mathias Bengtsson, $B S c^{a}$ Anna Eringfält, $B S c^{a}$ Jan Ernerudh, $M D, P h D^{a}$ Jenny Mjösberg, $P h D^{b}$ Maria C. Jenmalm, $P h D^{a}$

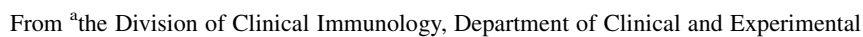
Medicine, Unit of Autoimmunity and Immune Regulation, Linköping University, Linköping, Sweden, and ${ }^{b}$ the Department of Medicine, Center for Infectious Medicine, Karolinska University Hospital Huddinge, Karolinska Institutet, Stockholm, Sweden. E-mail: anna.forsberg@liu.se.

The study was supported by grants from the Swedish Research Council (grant no. K2011-56X-21854-01-06), the Ekhaga Foundation, the Olle Engkvist Foundation, the Cancer and Allergy Association, and the University Hospital of Linköping, Sweden.

Disclosure of potential conflict of interest: J. Mjösberg has received research support from the Swedish Research Council and the Swedish Cancer Society. The rest of the authors declare that they have no relevant conflicts of interest.

\section{REFERENCES}

1. Spits H, Artis D, Colonna M, Diefenbach A, Di Santo JP, Eberl G, et al. Innate lymphoid cells-a proposal for uniform nomenclature. Nat Rev Immunol 2013; $13: 145-9$. 
2. Mjösberg JM, Trifari S, Crellin NK, Peters CP, van Drunen CM, Piet B, et al. Human IL-25- and IL-33-responsive type 2 innate lymphoid cells are defined by expression of CRTH2 and CD161. Nat Immunol 2011;12:1055-62.

3. Mjösberg J, Bernink J, Golebski K, Karrich JJ, Peters CP, Blom B, et al. The transcription factor GATA3 is essential for the function of human type 2 innate lymphoid cells. Immunity 2012;37:649-59.

4. Jenmalm MC. Childhood immune maturation and allergy development: regulation by maternal immunity and microbial exposure. Am J Reprod Immunol 2011;66: $75-80$

5. Abrahamsson TR, Sandberg Abelius M, Forsberg A, Björkstén B, Jenmalm MC A Th1/Th2-associated chemokine imbalance during infancy in children developing eczema, wheeze and sensitization. Clin Exp Allergy 2011;41 1729-39.

6. Chen W, Mempel M, Schober W, Behrendt H, Ring J. Gender difference, sex hormones, and immediate type hypersensitivity reactions. Allergy 2008;63:1418-27.

7. Casimir GJ, Mulier S, Hanssens L, Zylberberg K, Duchateau J. Gender differences in inflammatory markers in children. Shock 2010;33:258-62.

8. Pennell LM, Galligan CL, Fish EN. Sex affects immunity. J Autoimmun 2012;38: J282-91.

9. Postma DS. Gender differences in asthma development and progression. Gend Med 2007;4:S133-46.

http://dx.doi.org/10.1016/j.jaci.2014.01.027 


\section{METHODS}

\section{Study group}

Volunteer pregnant women were recruited from the maternal health care unit in Linköping. The children, 14 males and 13 females, were born in a period from August 2000 to March 2003. Only 1 of the children was delivered by cesarean section. Both parents signed an informed consent before the children's inclusion. The Regional Ethics Committee for Human Research at the University Hospital of Linköping approved the study (Dnr 99184 and 99323).

Seven of the children developed allergic symptoms and sensitization (a positive SPT result and/or detectable $\operatorname{IgE}$ to allergens) during the first 6 years of life (Table I) and 7 children remained healthy without sensitization. The remaining children developed either allergic symptoms without sensitization $(n=4)$ or sensitization without allergic symptoms $(n=4)$, while 5 children were not followed up because of various reasons. Because these 13 children cannot be definitely classified, they were not included in the allergy comparisons.

The children were monitored by research nurses at 6 and 12 months and follow-ups were done at 2 and 6 years by a pediatric allergologist. The parents answered questionnaires about environmental factors and allergic symptoms at 3, 6, 12, and 18 months and at 2 and 6 years.

Symptomatic diagnoses were set depending on predefined criteria. Atopic dermatitis was defined as chronic, pruritic, noninfectious dermatitis with typical appearance and anatomical localization. Urticaria was defined as an immediate skin reaction caused by the same allergen within an hour at least 2 times. Asthma was defined as bronchial obstruction with wheezing at least 3 times in total, at least 1 of these times diagnosed by a physician. Allergic rhinoconjunctivitis was defined as rhinitis and conjunctivitis appearing at least twice after exposure to an inhalant allergen and not related to infection. Food allergy was defined as vomiting and/or diarrhea on at least 2 separate occasions after the intake of a certain offending food. Of the 7 allergic children, 6 had atopic dermatitis, 3 had asthma, and 3 had rhinoconjunctivitis.

Skin prick tests were done on the volar aspect of the forearm. At the age of 6 months, fresh cow's milk (lipid concentration 0.5\%) and thawed egg white were used; at 12 months, milk, egg white, and cat extract (Allergologisk Laboratorium A/S [ALK], Soluprick, Hørsholm, Denmark) were included; and at 2 and 6 years, birch and timothy extracts (ALK) were added. Histamine hydrochloride $(10 \mathrm{mg} / \mathrm{mL}$ ) was used as positive control, and albumin diluent (ALK) was used as a negative control. If an allergen caused a wheal with a diameter of at least 3 millimeter, the test result was regarded as positive.

Sensitization was also measured through the analysis of circulating $\mathrm{IgE}$ antibodies to allergens. Levels of IgE antibodies to food antigens including egg, milk, fish, wheat, peanut, and soybean were tested with the PhadiatopInfant test (Phadia, Uppsala, Sweden) at ages 6, 12, and 24 months and 6 years. The Phadiatop test (Phadia) was used at 6 years to detect IgE antibodies to inhalant antigens birch, mugwort, timothy, cat, dog, horse, house-dust mite, and Cladosporium.

Volunteer adult individuals were recruited ( 9 men and 8 women, mean age, 34.9 and 35.1 years, respectively).

\section{Sample preparations}

Cord and adult peripheral blood was collected into heparinized vacutainers. Cord and adult PBMCs were obtained by Ficoll gradient centrifugation. Briefly, blood was layered on a Ficoll gradient, centrifuged, and the PBMC layer was collected with subsequently washing and centrifugation steps. Cells were resuspended in freezing media consisting of $40 \%$ Iscove's modified Dulbecco's medium, 10\% dimethyl sulfoxide, and 50\% FCS. Cells were then placed in a freezing container at $-70^{\circ} \mathrm{C}$ for 24 hours and thereafter stored in liquid nitrogen, pending analysis.

\section{Identification and characterization of cord and peripheral blood ILC2}

To explore the presence of ILC2, flow cytometry was used to analyze peripheral and cord blood. To obtain a reliable number of cells for analysis, 3 million cells were used for staining (cord blood mononuclear cell/PBMC) and 1 million lymphocytes were collected on the flow cytometer using forward scatter/side scatter. A lineage-negative population was identified; the antibody cocktail included the following antibodies (clone name within parentheses): fluorescein isothiocyanate-conjugated anti-CD1a (HI149), CD3 (OKT3), CD11c (3.9), CD123 (6H6), FCeR1 $\alpha$ (AER-37), TCR $\alpha \beta$ (IP26) (all from BioLegend, San Diego, Calif); CD4 (RPA-T4), CD14 (MфP9), CD19 (HIB19), CD34 (581), and TCR $\gamma \delta$ (B1) (all from Beckton Dickonson, Franklin Lakes, NJ); and CD303 (AC144, Miltenyi, Bergisch Gladbach, Germany). The low side scatter population expressed PECy7-conjugated antiCD127 (R34.34, Beckman Coulter, Brea, Calif), phycoerythrin-conjugated anti-CD161 (HP-3G10, BioLegend), and allophycocyanin-conjugated antiCD294 (BM16, BD Pharmingen, Franklin Lakes, NJ), and was partially positive for PerCpCy5.5-conjugated anti-CD117 (104D2, BioLegend) as compared with natural killer cells (APCCy7-conjugated anti-CD56 [HCD56], BioLegend). Cells were also stained with phycoerythrin-conjugated antiGATA3 (TWAJ, eBiosciences, San Diego, Calif) according to the manufacturer's instructions. Data were acquired on a BD FACS CANTO II and analyzed using Kaluzaa 1.2 (Beckman Coulter).

The effect of freezing was evaluated on peripheral blood from 6 individuals. The proportion of ILC2 was not affected by the freezing procedure (data not shown). However, the CD117 expression was significantly decreased after freezing and thawing $(P=.003$, data not shown). Because the proportion of ILC2 was unaffected by the freeze-thawing procedure, we used freeze-thawed samples from the birth cohort throughout this study. Also, the PBMCs from adults were frozen to limit variations between comparisons with CBMCs.

\section{Statistics}

Data are means \pm SD unless indicated otherwise. Statistical significance was examined by unpaired Student $t$ test. Statistical analyses were performed with GraphPad Prism software v5.0. 

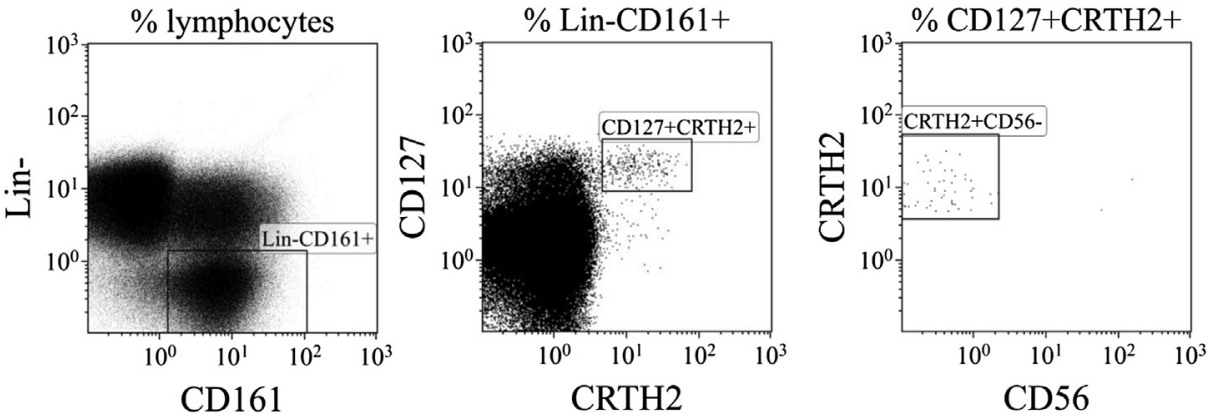

FIG E1. Gating strategy for ILC2 in human adult peripheral blood. To obtain a reliable number of cells for analysis, 3 million PBMCs were used for flow cytometry staining and 1 million lymphocytes were collected on the flow cytometer using forward scatter/side scatter. A lineage-negative population expressing CD161 was identified that also expressed CD127 and CRTH2 but was negative for CD56. 